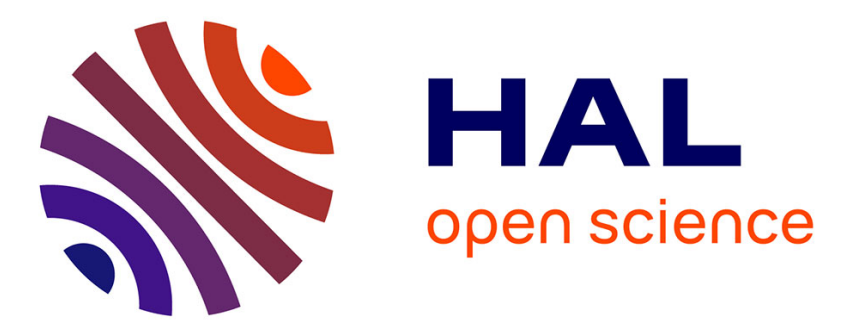

\title{
Calcul de propriétés thermodynamiques et des coefficients de transport dans un plasma Ar-O2 en non-équilibre thermodynamique et à la pression atmosphérique
}

\author{
J. Aubreton, C. Bonnefoi, J.M. Mexmain
}

\section{To cite this version:}

J. Aubreton, C. Bonnefoi, J.M. Mexmain. Calcul de propriétés thermodynamiques et des coefficients de transport dans un plasma Ar-O2 en non-équilibre thermodynamique et à la pression atmosphérique. Revue de Physique Appliquée, 1986, 21 (6), pp.365-376. 10.1051/rphysap:01986002106036500 . jpa00245455

\section{HAL Id: jpa-00245455 \\ https://hal.science/jpa-00245455}

Submitted on 1 Jan 1986

HAL is a multi-disciplinary open access archive for the deposit and dissemination of scientific research documents, whether they are published or not. The documents may come from teaching and research institutions in France or abroad, or from public or private research centers.
L'archive ouverte pluridisciplinaire HAL, est destinée au dépôt et à la diffusion de documents scientifiques de niveau recherche, publiés ou non, émanant des établissements d'enseignement et de recherche français ou étrangers, des laboratoires publics ou privés. 


\title{
Calcul de propriétés thermodynamiques et des coefficients de transport dans un plasma $\mathrm{Ar}-\mathrm{O}_{2}$ en non-équilibre thermodynamique et à la pression atmosphérique
}

\author{
J. Aubreton, C. Bonnefoi et J. M. Mexmain \\ C.N.R.S. LA 320, Equipe Thermodynamique et Plasmas, Université de Limoges, 123, avenue Albert Thomas, \\ 87060 Limoges Cedex, France
}

(Reçu le 21 décembre 1984, révisé le 11 février 1986, accepté le 21 février 1986)

\begin{abstract}
Résumé. - La composition du plasma thermique hors équilibre thermodynamique est calculée par une méthode statistique que nous avons appliqué aux plasmas de mélange argon-oxygène. Notre formalisme de calcul des coefficients de transport, pour ces plasmas, utilisent la définition classique des intégrales de collision. Pour leurs déterminations, nous avons donc sélectionné dans la littérature les potentiels d'interaction ou les sections efficaces de transfert (moment et charge) nécessaires. Les propriétés de transport (viscosité, conductivité électrique et conductibilité thermique) sont calculées :

- pour cinq valeurs du rapport $\mathrm{O}_{2} /\left(\mathrm{O}_{2}+\right.$ Ar) de l'oxygène pur à l'argon pur,

- pour $T_{\mathrm{e}}$ (température électronique) évoluant de $2000 \mathrm{~K}$ à $15000 \mathrm{~K}$,

- pour cinq valeurs du rapport $\theta=T_{\mathrm{e}} / T_{\mathrm{h}}\left(T_{\mathrm{h}}\right.$ température des particules lourdes) : $\theta=1$ (équilibre), 1,5, 2, 3 et 4 ,

- pour la pression atmosphérique.

Finalement, nos résultats sont comparés avec ceux de la littérature.

Abstracts. - For a thermal non-equilibrium Ar- $\mathrm{O}_{2}$ plasma, we compute the numerical densities by a statistical method. Our formalism of transport coefficients, for these kind of plasmas, uses the classical collision integrals. Therefore the collision integrals are determined from selected interaction potentials or from transfer cross-sections (charge and momentum). The transport properties (viscosity, electrical and thermal conductivity) are calculated :

- for five values of the $\mathrm{O}_{2} /\left(\mathrm{O}_{2}+\mathrm{Ar}\right)$ ratio, from pure oxygen to pure argon plasma,

- for $T_{\mathrm{e}}\left(T_{\mathrm{e}}\right.$ is the electronic temperature) varying from $2000 \mathrm{~K}$ to $15000 \mathrm{~K}$ by step of $100 \mathrm{~K}$,

- for five values of $\theta=T_{\mathrm{e}} / T_{\mathrm{h}}\left(T_{\mathrm{h}}\right.$ is the heavy particle temperature), $\theta=1$ (equilibrium), 1.5, 2, 3 and 4.

- for the atmospheric pressure.

At last, our results are compared to those of the literature.
\end{abstract}

\section{Introduction.}

Toute modélisation du milieu plasma (tel que interaction plasma-surface, écoulement, ...) nécessite la connaissance de ses propriétés de transport. Dans les plasmas « hautes pressions " $(P>100$ torrs), lorsque le degré d'ionisation est suffisant (ce qui assure un couplage entre les électrons et les particules lourdes par l'intermédiaire des ions) et si les grandeurs macroscopiques (températures, densités) évoluent peu sur des longueurs très grandes vis-à-vis des longueurs macroscopiques qui caractérisent le milieu (longueur de Debye, libre parcours moyen, ...) on fait l'hypothèse raisonnable de l'équilibre thermodynamique local
(ETL). On emploie alors des méthodes de calcul désormais classiques : minimisation de l'énergie libre pour la détermination de la composition du gaz plasmagène et pour les propriétés de transport les méthodes décrites dans les références [1-4] à la condition que les sections efficaces de collision élastique ou les potentiels d'interaction entre les diverses espèces présentes dans le système soient connus. Cette hypothèse d'ETL est cependant souvent mise en défaut; en effet, de nombreuses études expérimentales [5] à [8] montrent, en particulier, que dans des plasmas en extinction et au voisinage d'une surface solide ou liquide, dans la couche limite, l'énergie cinétique moyenne des électrons est, le plus souvent, supé- 
rieure à celles des particules lourdes. Il y a alors redistribution des vitesses pour ces deux sous-ensembles de particules, au voisinage de deux maxwelliennes, l'une à la température $T_{\mathrm{e}}$ pour les électrons et l'autre à la température $T_{\mathrm{h}}$ pour les particules lourdes. Dans deux précédents articles nous avons adopté la théorie cinétique de Chapman-Enskog à un plasma à deux températures et nous en avons déduit les expressions des coefficients de transport jusqu'à la quatrième approximation pour les processus élastiques [9] et à la première approximation pour les processus inélastiques [10].

Dans ce travail, nous appliquons les résultats de $[9,10]$ au plasma de mélange argon-oxygène, sans revoir le formalisme lié à la détermination des propriétés de transport. Rappelons simplement que le calcul de la composition est basé sur une méthode dérivée de celle de Potapov [11] et que nous avons déjà utilisée pour un plasma de mélange argonhydrogène [12].

Dans la première partie $(\S 2)$ nous développons l'étude de tous les types de collision intervenant dans le plasma de mélange : potentiels d'interaction (les intégrales de collision sont alors calculées à l'aide de tables) et sections efficaces de transfert. Les résultats sont présentés au $\S 3$ pour différentes valeurs de la composition du mélange. L'enthalpie massique, la chaleur spécifique massique à pression constante, la viscosité, la conductibilité thermique et la conductivité électrique sont calculées pour des valeurs de la température des électrons comprise entre $2000 \mathrm{~K}$ et $15000 \mathrm{~K}$, et pour $\theta=1 ; 1,5 ; 2 ; 3 ; 4\left(\theta=T_{\mathrm{e}} / T_{\mathrm{h}}\right)$. Enfin nous discutons les résultats et nous les comparons à ceux proposés dans la littérature.

\section{Intégrales de collision. Potentiel d'interaction.}

A la pression atmosphérique et pour une température maximale de $15000 \mathrm{~K}$, nous faisons l'hypothèse que le plasma est constitué uniquement des espèces suivantes :

$$
\mathrm{Ar}, \mathrm{Ar}^{+}, \mathrm{O}_{2}, \mathrm{O}_{2}^{+}, \mathrm{O}, \mathrm{O}^{+}, \mathrm{O}^{-} \text {et e . }
$$

Les propriétés de transport sont, dans notre formalisme $[9,10]$, exprimées en fonction des intégrales de collision classique

$$
\begin{aligned}
\bar{\Omega}^{(l, s)}=\frac{\bar{Q}^{(l, s)}}{\pi}= & \frac{2(l+1)}{\pi(s+1) !\left[2 l+1+(-1)^{l}\right]} \\
& \times \int_{0}^{\infty} X^{s+1} \exp (-X) Q^{l}(X) \mathrm{d} X
\end{aligned}
$$

où

$$
X=\frac{\varepsilon}{k T}
$$

$\varepsilon$ étant l'énergie relative des particules, $k$ la constante de Boltzmann et $T$ la température où $Q^{l}(X)$ est une section efficace de transfert. Le niveau d'approximation retenu dans le développement de Sonine fixe le nombre de couples $(l, s)$ nécessaire c'est-à-dire le nombre d'intégrales de collision à calculer. L'obtention de ces intégrales de collision se fait alors par deux méthodes :

- soit la section efficace de transfert $Q^{l}$ est connue (expérimentalement ou théoriquement) et l'on intègre numériquement la relation (1)

- soit le potentiel d'interaction lors d'une collision (relié directement à $Q^{l}$ ) est connu, la détermination de $\bar{\Omega}^{(l, s)}$ passe alors par des tables.

Nous allons voir successivement les collisions chargée-chargée, neutre-neutre, neutre-chargée.

2.1 InTERACTION CHARGÉE-CHARGÉE. - Lors d'une collision du type ion-ion et électron-ion le potentiel d'interaction interparticulaire $V(r)$ est de type Coulombien avec écran, il s'écrit :

$$
V(r)=\frac{z_{i} z_{j} e^{2}}{4 \pi \varepsilon_{0}} \exp (-r / d)
$$

où $z_{k}$ est le nombre de charge de l'espèce $k, e$ est la charge de l'électron, $\varepsilon_{0}$ est la permittivité du vide et $d$ la longueur de Debye.

Les temps d'interactions étant brefs (à l'intérieur de la sphère de Debye) on fait l'hypothèse de la quasiimmobilité ionique c'est-à-dire que l'on prend en compte uniquement la contribution électronique et l'on écrit :

$$
d=\left(\frac{\varepsilon_{0} k T_{\mathrm{e}}}{n_{\mathrm{e}} e^{2}}\right)^{1 / 2}
$$

où $n_{\mathrm{e}}$ est la densité électronique.

Les intégrales de collision sont calculées à partir des tables de Mason et al. [13] complétées par celles de Devoto [14].

2.2 INTERACTION NEUTRE-NEUTRE. - Dans la littérature, on trouve un grand nombre de formes analytiques de potentiel interparticulaire pour ce type de collision. Nous rappelons ici uniquement les formes analytiques qui nous ont servi dans cette étude.

Les potentiels puissance (noté $P$ dans les tableaux) :

$$
V(r)= \pm V_{0} r^{-n}
$$

Les intégrales de collision correspondantes sont calculées à l'aide des tables de Kihara et al. [15].

La forme exponentielle (noté E dans les tableaux) :

$$
V(r)= \pm V_{0} \exp (-B r)
$$

Les intégrales de collision correspondantes sont obtenues à partir des tables de Monchick [16] (potentiel répulsif) et des tables de Brokaw [17] (potentiel attractif).

Le potentiel de Morse [18] (noté $M$ dans les ta- 
bleaux) :

$$
V(r)=D_{\mathrm{e}}\left[\exp \left(-2 \beta\left(r-r_{\mathrm{m}}\right)\right)-2 \exp \left(-\beta\left(r-r_{\mathrm{m}}\right)\right)\right]
$$

où $D_{\mathrm{e}}$ est la profondeur du puits de potentiel et $r_{\mathrm{m}}$ la distance interparticulaire correspondant à l'équilibre (c'est-à-dire correspondant au minimum de $V(r)$ ).

Les intégrales de collisions sont calculées à partir des tables de Smith et al. [19].

Le potentiel de Buckimgham [20, 21] (noté $B$ dans les tableaux)

$$
V(r)=\frac{D_{\mathrm{e}}}{\alpha-6}\left[6 \exp \left(\alpha\left(1-\frac{r}{r_{\mathrm{m}}}\right)\right)-\alpha\left(\frac{r_{\mathrm{m}}}{r}\right)^{6}\right]
$$

Les intégrales de collision correspondantes sont calculées à l'aide des tables de Mason [21].

Dans le tableau I, nous donnons les coefficients des potentiels d'interaction que nous avons utilisés pour le calcul des intégrales de collision (interaction neutreneutre).

2.3 INTERACTION NEUTRE-ION. - Les intégrales de collision se calculent comme dans les cas des inter- actions neutre-neutre, à l'exception de celles de type diffusion $(l=1)$ lors de collision du type $M \cdot M^{+}$ (transfert résonnant) [28]. Il faut alors connaître la section efficace de transfert de charge $Q_{\text {tr. }}$ que l'on écrit sous la forme suivante [29] (noté $T$ dans le tableau II).

$$
Q_{\mathrm{tr} .}=\frac{1}{2}(A-B \ln (g))^{1 / 2}
$$

où $I$ et $B$ sont des coefficients obtenus expérimentalement ou théoriquement et où $g$ est la vitesse relative de collision. Connaissant $A$ et $B$, les intégrales de collision sont alors facilement calculées [30].

Dans le tableau II, nous donnons, soit les coefficients des potentiels d'interaction (les notations sont celles du tableau I), soit les coefficients $A$ et $B$, qui nous ont été nécessaires pour le calcul des intégrales de collision (interaction neutre-ion).

2.4 INTERACTION NEUTRE-ÉleCTRON. - Nous calculons les intégrales de collision $(l=1)$ par intégration numérique (méthode de Gauss-Laguerre), à partir de la relation de définition des intégrales de collision $\bar{Q}^{(l, s)}($ relation (1)). Pour les interactions e-Ar, e-O et e- $\mathrm{O}_{2}$ nous utilisons les sections efficaces de transfert de moment d'Itikawa [37, 38].

Tableau I. - Valeurs des paramètres des potentiels d'interaction pour les collisions neutre-neutre.

[Values of the parameters for the interaction potentials used in neutral-neutral collisions.]

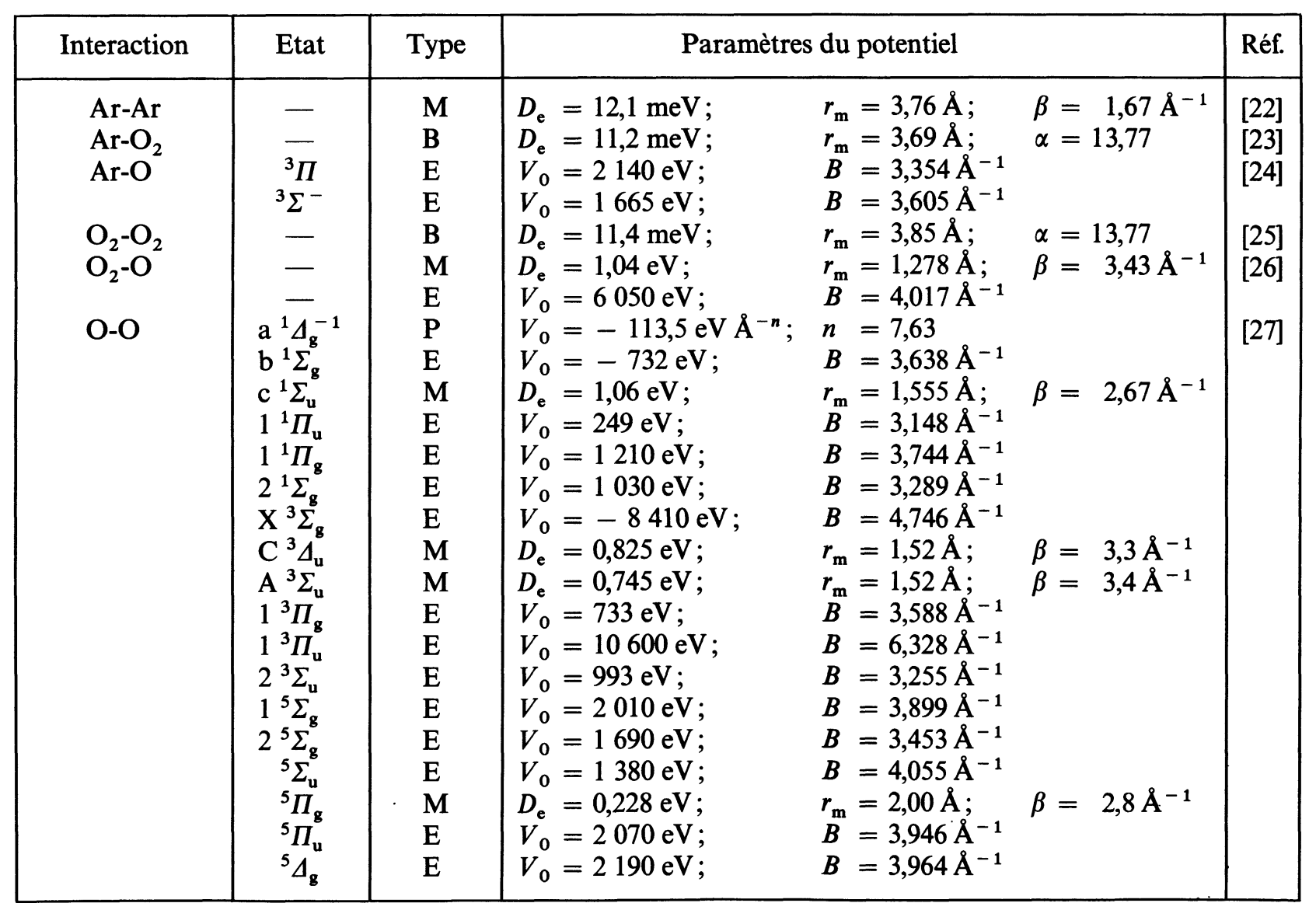


Tableau II. - Valeurs des paramètres de potentiels d'interaction et des coefficients des sections efficaces de transfert de charge (relation (8)) pour les collisions ion-neutre.

[Values of the parameters for the interaction potentials and of the coefficients for the charge transfer crosssections (relation (8)) used in ion-neutral collisions.]

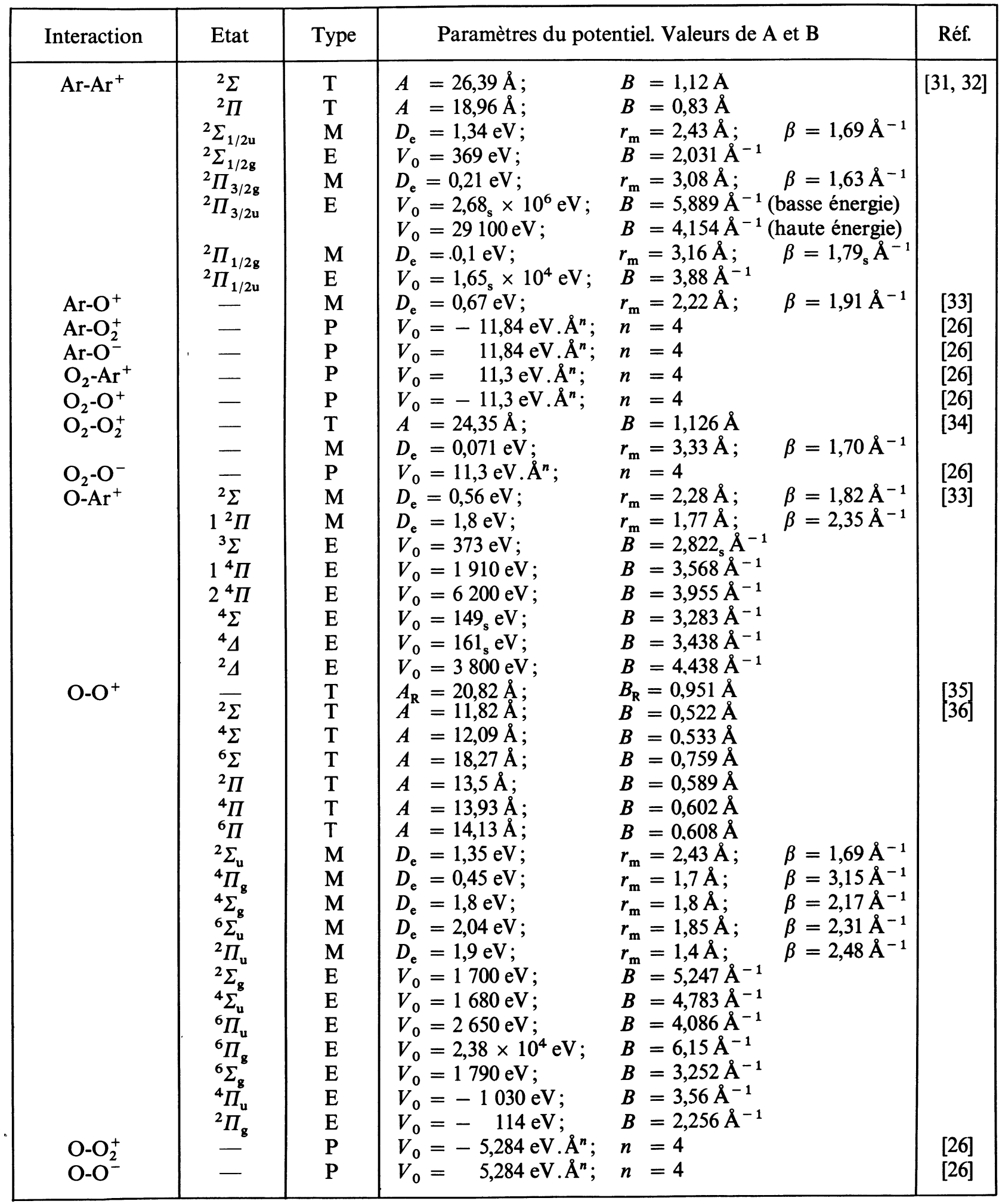




\section{Résultats.}

3.1 Composition. PropriÉtÉs THERMOdYNAMiQues. La composition du mélange argon-oxygène est calculée par une méthode MSE (Modified Saha Equations) analogue à celle décrite par Potapov [11] présentée dans la référence [12]. Bien que les résultats obtenus à partir de cette théorie soient discutables [39], elle présente le grand avantage de nécessiter uniquement la connaissance des fonctions de partition interne des diverses espèces. Celles-ci dépendent $a$ priori des deux valeurs des températures $T_{\mathrm{e}}$ et $T_{\mathrm{h}}$ mais aussi de la composition du plasma au travers des théories de limitation. Nous avons fait les hypothèses suivantes pour les fonctions de partition interne :

- elles sont indépendantes de la composition et nous utilisons celles de Capitelli et al. [40] que nous complétons jusqu'à $500 \mathrm{~K}$ grâce aux tables de la JANAF.

- elles ne dépendent, pour les atomes et leurs ions que de la température électronique $T_{\mathrm{e}}$ et pour les molécules et leurs ions que de la température des particules lourdes $T_{\mathrm{h}}$ [12].

Pour des raisons de facilités de calcul, les fonctions de partition interne ainsi que leurs dérivées première et seconde sont introduites dans nos programmes sous la forme de polynôme d'ajustement (il en est de même pour les intégrales de collision).

La composition du mélange est calculée à la pression atmosphérique, pour $T_{\mathrm{e}}$ variant de $2000 \mathrm{~K}$ à $15000 \mathrm{~K}$ par pas de $100 \mathrm{~K}$ et ce pour cinq valeurs du rapport $\theta=T_{\mathrm{e}} / T_{\mathrm{h}}(\theta=1 ; 1,5 ; 2 ; 3 ; 4)$ et pour cinq valeurs du rapport de concentrations $\mathrm{O}_{2} /\left(\mathrm{O}_{2}+\mathrm{Ar}\right)$ : de l'oxygène pur à l'argon pur par pas de $25 \%$. Les résultats obtenus sont en très bon accord avec ceux de Capitelli et al. [40] (à moins de $1 \%$ ) dans le cas de l'équilibre. Sur les figures 1 à 3 sont représentées les valeurs des densités numériques des espèces de plasma, en fonction de la température électronique $T_{\mathrm{e}}$, dans le cas de l'argon pur (Fig. 1) et du mélange à $50 \%$

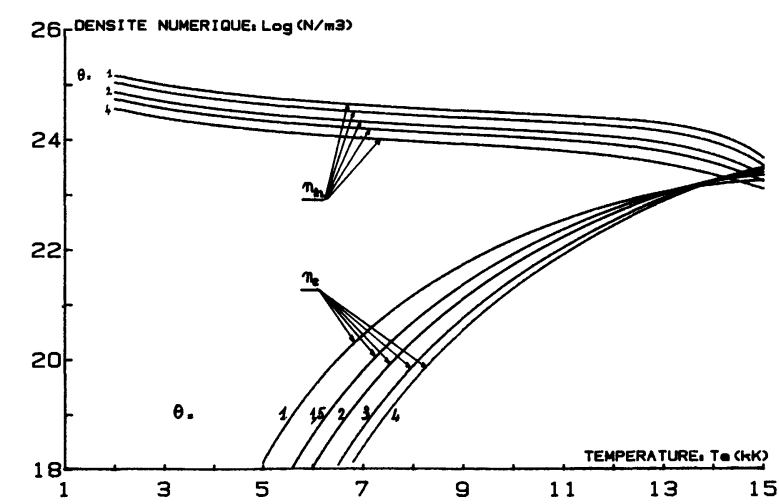

Fig. 1. - Evolution des densités numériques, en fonction de la température $T_{\mathrm{e}}$ et pour $\theta=1,1,5,2,3$ et 4 , du plasma d'argon pur à la pression atmosphérique.

[Evolution, with electronic temperature and $\theta=1, \ldots, 4$, of the numerical densities for an argon plasma $(p=1 \mathrm{~atm})$.] d'oxygène pour $\theta=1$ et 2 (Figs. 2 et 3 ). Et sur la figure 4 , nous représentons les valeurs des densités numériques des électrons, en fonction de $T_{\mathrm{e}}$, dans le cas du mélange à $50 \%$ d'oxygène et pour $\theta=1,1,5,2$ et 4 .

Les propriétés thermodynamiques que nous calculons sont l'enthalpie massique $\hat{h}$ définie comme suit :

$$
\hat{h}=\frac{\sum_{i=1}^{N} n_{i} h_{i}}{\sum_{i=1}^{N} n_{i} m_{i}}
$$

où $n_{i}, h_{i}, m_{i}$ sont, respectivement, la densité numérique, l'enthalpie et la masse de la particule de l'espèce $i$.

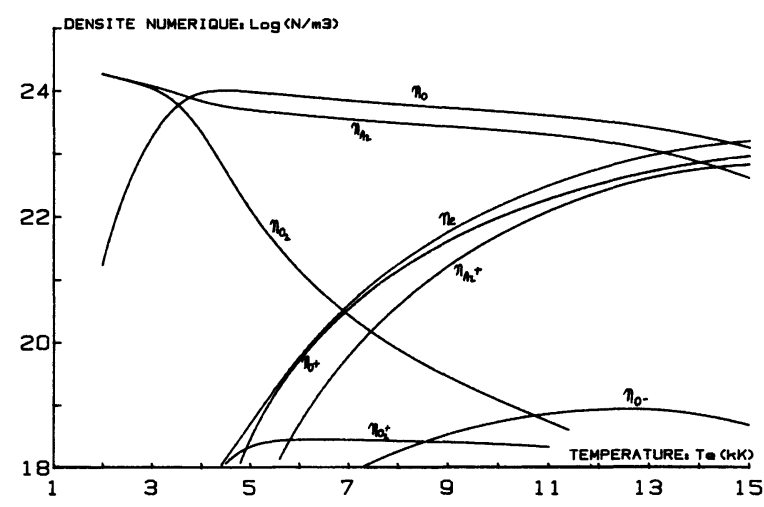

Fig. 2. - Evolution des densités numériques, en fonction de la température électronique $T_{\mathrm{e}}$ et à l'équilibre $(\theta=1)$, d'un plasma de mélange à $50 \%$ de $\mathrm{O}_{2}$ à la pression atmosphérique.

[Evolution, with electronic temperature of the numerical densities for an argon-oxygen plasma $\left(50 \%\right.$ of $\left.\mathrm{O}_{2}\right)$ : atmospheric pressure and $\theta=1$.]

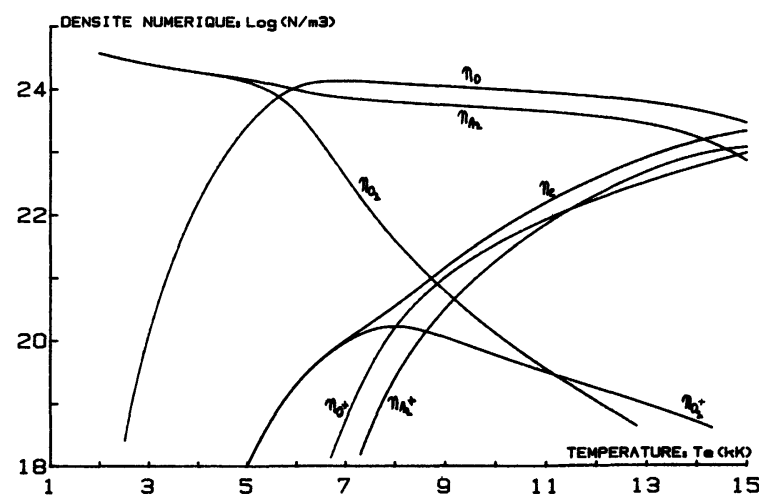

Fig. 3. - Evolution des densités numériques, en fonction de la température $T_{\mathrm{e}}$ et pour $\theta=2$, d'un plasma de mélange à $50 \%$ de $\mathrm{O}_{2}$ à la pression atmosphérique.

[Evolution, with electronic temperature, of the numerical densities for an argon-oxygen plasma $\left(50 \%\right.$ of $\left.\mathrm{O}_{2}\right)$ : atmospheric pressure and $\theta=2$.] 


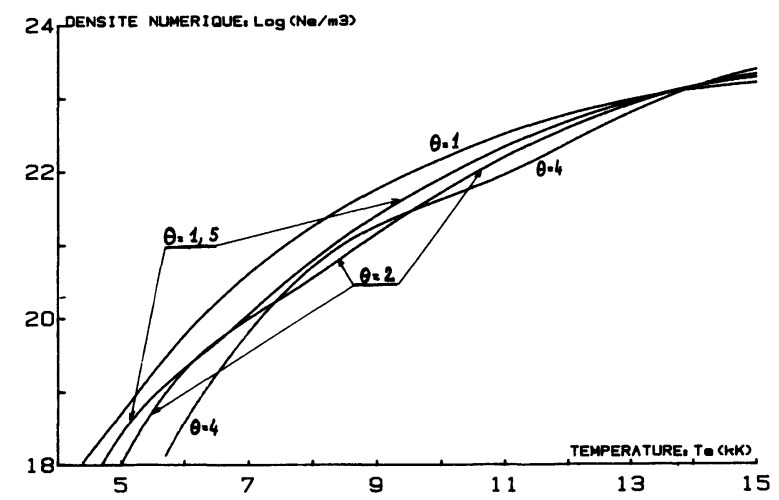

Fig. 4. - Evolution des densités numériques des électrons en fonction de la température électronique $T_{\mathrm{e}}$ et pour $\theta=1,1,5,2$ et 4 , d'un plasma de mélange à $50 \%$ de $\mathrm{O}_{2}$ à la pression atmosphérique.

[Evolution, with electronic temperature and $\theta=1, \ldots, 4$, of the electronic numerical densities for an argon-oxygen plasma $\left(50 \%\right.$ of $\left.\mathrm{O}_{2}\right)$ : atmospheric pressure.]

Puis nous calculons la chaleur spécifique massique à pression constante par simple dérivation numérique à cinq points de l'enthalpie massique :

$$
\hat{C}_{p}=\left(\frac{\partial \hat{h}}{\partial T_{\mathrm{e}}}\right)_{p}
$$

Rappelons cependant que $\hat{C}_{p}$ est la somme de deux composantes qui apparaissent de manière évidente lors de la dérivation, par rapport à $T_{\mathrm{e}}$, de la relation (9) :

$$
\hat{C}_{p}=\hat{C}_{p}(\text { Fig. })+\hat{C}_{p} \text { (Réac.) } .
$$

La première est dite figée, elle correspond à la somme sur les $C_{p}^{i}=\left(\frac{\partial h_{i}}{\partial T_{\mathrm{e}}}\right)_{p}$. La deuxième est dite réactionnelle, elle fait intervenir les $\left(\frac{\partial n_{i}}{\partial T_{\mathrm{e}}}\right)_{p}$ donc tient compte des réactions chimiques.

Les valeurs de ces deux grandeurs : $\hat{h}$ et $\hat{C}_{p}$ sont représentées en fonction de la température électronique $T_{\mathrm{e}}$ et pour le mélange à $50 \%$ d'oxygène, sur les figures 5 et 6 respectivement. Sur la figure 6 les pics de gauche de $\hat{C}_{p}\left(T_{\mathrm{e}} \# 4000\right.$ pour $\theta=1, T_{\mathrm{e}}=$ $5000 \mathrm{~K}$ pour $\theta=1,5, T_{\mathrm{e}} \# 6000 \mathrm{~K}$ pour $0=2$, $T_{\mathrm{e}} \# 7500 \mathrm{~K}$ pour $\theta=3$ et $T_{\mathrm{e}} \# 8500 \mathrm{~K}$ pour $\theta=4$ ) correspondent à la réaction de dissociation de l'oxygène moléculaire et les pics de droite à la réaction d'ionisation de l'argon et de l'oxygène atomique.

3.2 PROPRIÉTÉS DE TRANSPORT. - Nous ne rappelons pas le formalisme permettant le calcul des propriétés de transport, nous nous référons aux articles $[9,10]$. Pour les intégrales de diffusion $(l=1)$ de la collision $\mathrm{O}-\mathrm{O}^{+}$nous notons (1) celles obtenues à

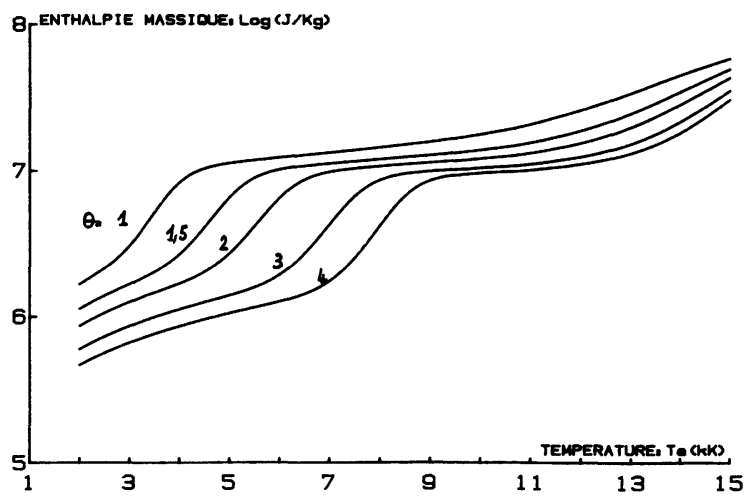

Fig. 5. - Evolution de l'enthalpie massique, en fonction de la température électronique $T_{\mathrm{e}}$ et pour $\theta=1,1,5,2,3$ et 4 , d'un plasma de mélange à $50 \%$ de $\mathrm{O}_{2}$ à la pression atmosphérique.

[Evolution, with electronic temperature and $\theta=1, \ldots, 4$, of the massic enthalpy for an argon-oxygen plasma $(50 \%$ of $\left.\mathrm{O}_{2}\right): p=1 \mathrm{~atm}$.]

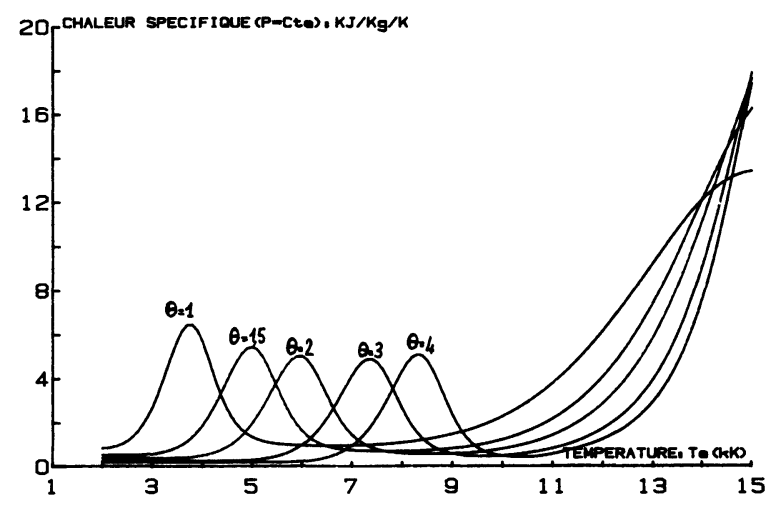

Fig. 6. - Evolution de la chaleur spécifique massique à pression constante en fonction de la température $T_{\mathrm{e}}$ et pour $\theta=1,1,5,2,3$ et 4 d'un plasma de mélange à $50 \%$ de $\mathrm{O}_{2}$ à la pression atmosphérique.

[Evolution, with electronic temperature and $\theta=1, \ldots, 4$, of the massic specific heat for an argon-oxygen plasma $(50 \%$ of $\left.\mathrm{O}_{2}\right): p=1 \mathrm{~atm}$.]

partir des résultats de Rutherford et al. [35] et nous notons (2) celles dérivées des travaux de Beebe $e t$ al. [36]. Lorsqu'aucune précision est donnée, ce sont les intégrales de la série (2) qui sont utilisées pour des raisons de cohérence avec les intégrales de viscosité $(l=2)$ : mêmes données de base.

3.2.1 Viscosité du gaz de particules lourdes. - Pour un calcul de la viscosité $\eta$ un développement au premier ordre de la méthode de Chapman-Enskog est suffisant [2]. Nous représentons sur les figures 7 et 8 , en fonction de la température électronique et pour $\theta=1 ; 1,5 ; 2 ; 3 ; 4$, la viscosité de l'argon pur et du mélange à $50 \%$ d'oxygène. Nous constatons que 


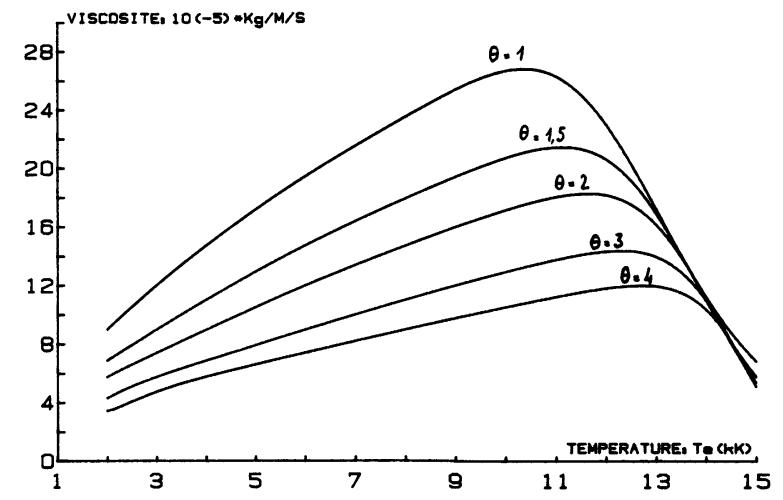

Fig. 7. - Evolution de la viscosité, en fonction de la température $T_{\mathrm{e}}$ et pour $\theta=1,1,5,2,3$ et 4, d'un plasma d'argon pur à la pression atmosphérique.

[Evolution, with electronic temperature and $\theta=1, \ldots, 4$, of the viscosity for an argon plasma $(p=1 \mathrm{~atm})$.]

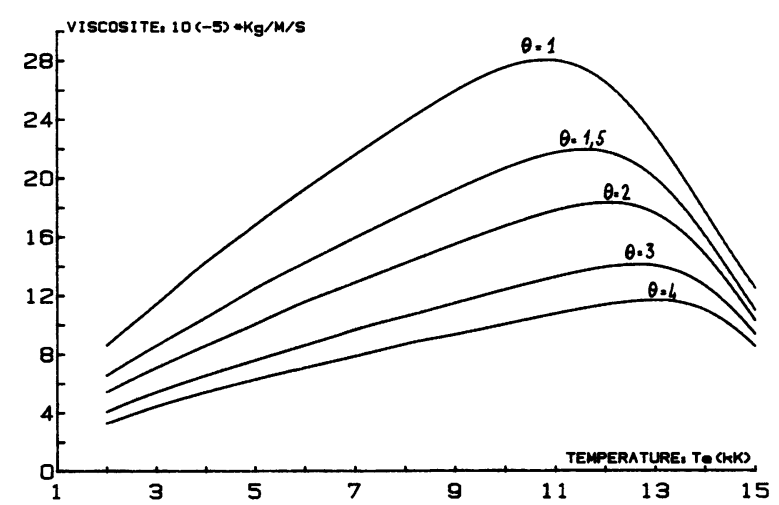

Fig. 8. - Evolution de la viscosité, en fonction de la température $T_{\mathrm{e}}$ et pour $\theta=1,1,5,2,3$ et 4 , d'un plasma de mélange à $50 \%$ de $\mathrm{O}_{2}$ à la pression atmosphérique.

[Evolution, with electronic temperature and $\theta=1, \ldots, 4$, of the viscosity for an argon-oxygen plasma $\left(50 \%\right.$ of $\left.\mathrm{O}_{2}\right)$ : $p=1$ atm. ]

l'allure générale des courbes est indépendante de la composition du plasma ainsi que du degré de déséquilibre. Le maximum du pic marque la limite entre les plasmas «dominés par les neutres " (aux basses températures) de ceux « régis par les ions " (aux hautes températures). En effet, les interactions ionion conduisent à des valeurs des intégrales de collision très grandes par rapport aux autres (neutre-neutre et ion-neutre). Ceci a pour conséquence de faire chuter la viscosité; $\Omega^{(2,2)}$ intervient au dénominateur de la relation permettant son calcul. Lorsque la valeur de $\theta$ augmente et quel que soit le mélange plasma, le sommet du pic de viscosité se déplace vers les hautes températures ce qui correspond à une apparition de plus en plus tardive des ions.

Pour l'argon pur nos résultats sont en bon accord avec ceux proposés par Kannapan et al. [41] et par Hsu et al. [42].
A l'équilibre $(\theta=1)$, la viscosité de l'oxygène pur est du même ordre de grandeur et même supérieure à celle de l'argon (quelle que soit la série d'intégrales de collision utilisées pour l'interaction O-O) contrairement à d'autres résultats proposés dans la littérature [43, 44]. Dans le tableau III, nous comparons la viscosité de l'oxygène pur (calculée pour les deux séries d'intégrales) à celle de l'argon pur (les résultats sont exprimés en $10^{-4} \mathrm{~kg} / \mathrm{m} / \mathrm{s}$ ) pour deux valeurs de la température $T_{\mathrm{e}}$.

Nous attribuons ce fait à ce que ces auteurs utilisent les intégrales de collision de Yun et al. [45], pour l'interaction $\mathrm{O}-\mathrm{O}$, qui diffèrent sensiblement des nôtres (basées sur des résultats plus récents).

Dans le tableau IV, nous comparons nos résultats (pour $\Omega^{(2,2)}$ en $\AA^{2}$ ) à ceux de Yun et al. [45] et à ceux de Capitelli et al. [46] qui conduiraient à des valeurs de la viscosité de l'oxygène encore plus élevées.

3.2.2 Conductivité électrique. - Pour la conductivité électrique $\sigma$ nous prenons en compte que les électrons. La méthode de Chapman-Enskog converge alors lentement, nous utilisons donc un développement au troisième ordre [3].

Sur les figures 9 et 10, nous représentons, respectivement, la conductivité électrique du plasma, en fonction de la température $T_{\mathrm{e}}$ et pour $\theta=1 ; 1,5 ; 2 ; 3 ; 4$, pour l'argon pur et pour le mélange à $50 \%$ d'oxygène.

Tableau III. - Comparaison des valeurs de la viscosité (exprimée en $10^{-4} \mathrm{~kg} / \mathrm{m} / \mathrm{s}$ ) et les nôtres obtenues à partir des données de Rutherford [35] (noté 1) et de celles de Beebe [36] (noté 2).

[Comparison of viscosity values (in $10^{-4} \mathrm{~kg} / \mathrm{m} / \mathrm{s}$ ) and ours obtained from Rutherford data [35] (noted (1)) and the ones of Beebe [36] (noted (2).]

\begin{tabular}{|c|c|c|c|c|}
\hline$T_{\mathrm{e}}(\mathrm{K})$ & $\mathrm{O}_{2}(1)$ & $\mathrm{O}_{2}(2)$ & $\mathrm{O}_{2}[43]$ & $\mathrm{Ar}$ \\
\hline 5000 & 1,670 & 1,670 & 1,55 & 1,717 \\
\hline 10000 & 2,773 & 2,791 & 2,46 & 2,660 \\
\hline
\end{tabular}

Tableau IV. - Comparaison des valeurs de $\bar{\Omega}^{(2,2)}$ (exprimée en $\AA^{2}$ ) pour la collision $\mathrm{O}-\mathrm{O}$, obtenues par Yunn [45], Capitelli [46] et nos résultats.

[Comparison between $\bar{\Omega}^{(2,2)}$ values, for the O-O collision, calculated by Yunn [45], Capitelli [46] and our data.]

$\begin{array}{rccc}T_{\mathrm{e}}(\mathrm{K}) & \bar{\Omega}^{(2,2)}[45] & \bar{\Omega}^{(2,2)}[46] & \text { Nos résultats } \\ - & - & - & - \\ 5000 & 4,88 & 4,41 & 4,53 \\ 10000 & & 3,67 & 3,70 \\ 14000 & & 3,34 & 3,34\end{array}$




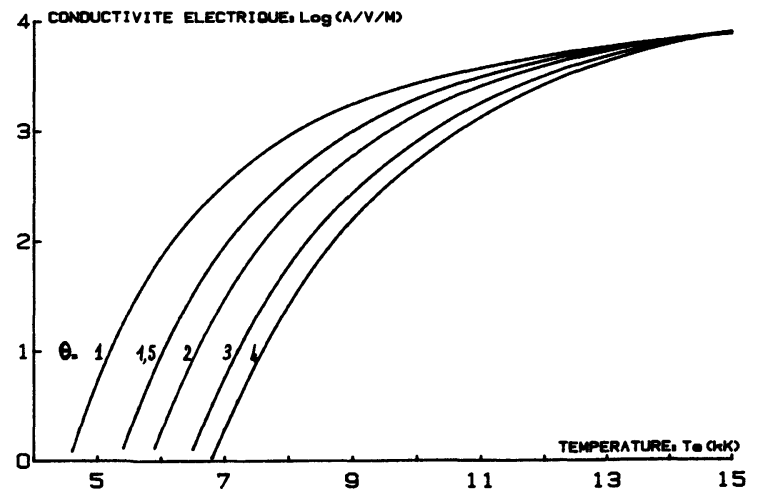

Fig. 9. - Evolution de la conductivité électrique, en fonction de la température $T_{\mathrm{e}}$ et pour $\theta=1,1,5,2,3$ et 4 , d'un plasma d'argon pur à la pression atmosphérique.

[Evolution, with electronic temperature and $\theta=1, \ldots, 4$, of the electrical conductivity for an $\operatorname{argon} \operatorname{plasma}(p=1 \mathrm{~atm})$.]

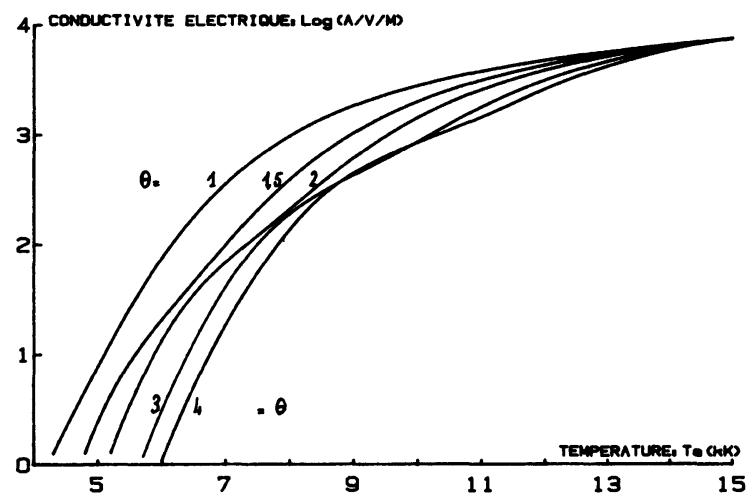

Fig. 10. - Evolution de la conductivité électrique, en fonction de la température $T_{\mathrm{e}}$ et pour $\theta=1,1,5,2,3$ et 4 , d'un plasma de mélange à $50 \% \mathrm{de}_{2}$ à la pression atmosphérique.

[Evolution, with electronic temperature and $\theta=1, \ldots, 4$, of the electrical conductivity for an argon-oxygen plasma $\left(50 \%\right.$ of $\left.\mathrm{O}_{2}\right): p=1 \mathrm{~atm}$.]

Pour l'argon pur, en comparant les figures 1 et 9 , nous remarquons une similitude entre les courbes représentant la densité numérique des électrons et lá conductivité électrique, et ce pour les différentes valeurs de $\theta$ : même position relative en fonction de $\theta$ et elles se rejoignent pour $T_{\mathrm{e}} \# 15000 \mathrm{~K}$. Ce parallèle met en évidence, comme on pouvait s'y attendre, le fait que les électrons jouent un rôle essentiel dans la conductivité électrique du plasma. Nos résultats sont en bon accord avec ceux de Kannapan et al. [41] et de Hsu et al. [42].

Pour le mélange à $50 \%$ d'oxygène (Fig. 10), nous constatons des ruptures de pente de la fonction $\sigma\left(T_{\mathrm{e}}\right)$ pour $\theta=1,5$ et $T_{\mathrm{e}} \# 6000 \mathrm{~K}, \theta=2$ et $T_{\mathrm{e}} \# 7500 \mathrm{~K}$ et $\theta=4$ et $T_{\mathrm{e}} \# 10500 \mathrm{~K}$. Ceci résulte directement de la variation de pente de la densité électronique $n_{\mathrm{e}}$ $n_{\mathrm{e}}\left(T_{\mathrm{e}}\right)$ (Fig. 4) et nous attribuons ce phénomène à une compétition entre diverses réactions. Nous prenons le cas de $\theta=2$ pour exemple. Aux basses températures, pour $T_{\mathrm{e}}<6000 \mathrm{~K}$ (ce qui correspond à $T_{\mathrm{h}}<$ $3000 \mathrm{~K}$ ) les électrons sont essentiellement produits par des processus d'ionisation de $\mathrm{O}_{2}$.

$$
\mathrm{O}_{2} \rightarrow \mathrm{O}_{2}^{+}+\mathrm{e} \text {. }
$$

Une réaction concurrente, pour la disparition de $\mathrm{O}_{2}$ est la dissociation :

$$
\mathrm{O}_{2} \rightarrow 2 \mathrm{O}
$$

réaction qui sera de plus en plus efficace avec l'augmentation de la température. Donc, pour $T_{\mathrm{e}} \# 7500 \mathrm{~K}$ $\left(T_{\mathrm{h}}=3800 \mathrm{~K}\right)$, la réaction $(\alpha)$ est inhibée par la réaction $(\beta)$ et la production d'électrons à partir des espèces atomiques (ionisation de $\mathrm{A}_{2}$ et de $\mathrm{O}$ ) est encore peu efficace, ce qui conduit, pour $\theta=2$ et $T_{\mathrm{e}} \# 7500 \mathrm{~K}$, à ce palier des courbes $\eta_{\mathrm{e}}=f\left(T_{\mathrm{e}}\right)$ et $\sigma=f\left(T_{\mathrm{e}}\right)$.

3.2.3 Conductibilité thermique. - La conductibilité thermique totale est la somme de quatre contributions

$$
\lambda_{\mathrm{T}}=\lambda_{\mathrm{h}}+\lambda_{\mathrm{e}}+\lambda_{\mathrm{R}}+\lambda_{\text {Int. }}
$$

Les deux premières, $\lambda_{\mathrm{h}}$ et $\lambda_{\mathrm{e}}$, sont liées aux transferts d'énergie lors d'une collision élastique. Collision entre particules lourdes $\lambda_{\mathrm{h}}$ (développement jusqu'à la deuxième approximation [3]) et collision faisant intervenir un électron $\lambda_{\mathrm{e}}$ (développement jusqu'à la troisième approximation [3]). Les deux dernières, $\lambda_{R}$ et $\lambda_{\text {Int. }}$ (développement jusqu'à la première approximation [1]), rendent compte des contributions dues aux transferts d'énergie lors de collisions réactives soit par réaction chimique $\lambda_{R}$, soit par échange d'énergie interne $\lambda_{\text {Int. }}$.

3.2.3.1 Influence des diverses contributions sur $\lambda_{\mathrm{T}}$. Sur la figure 11 nous représentons la conductibilité thermique totale ainsi que ses composantes, en fonction de la température $T_{e}$, pour un mélange à $75 \%$ d'oxygène et pour $\theta=2$. Cette figure montre l'influence respective des quatre contributions :

$-\lambda_{\mathrm{h}}$ varie peu sur la plage de température considérée, sa contribution à $\lambda_{\mathrm{T}}$ est toujours de l'ordre de $0,25 \mathrm{~W} / \mathrm{m} / \mathrm{K}$.

- $\lambda_{\mathrm{e}}$ est importante qu'à haute température, à $15000 \mathrm{~K}$ où sa contribution est maximale, elle représente $25 \%$ de $\lambda_{T}$.

- $\lambda_{\mathrm{h}}$ qui donne la forme de la fonction $\lambda_{\mathrm{T}}=f\left(T_{\mathrm{e}}\right)$ présente deux pics, l'un à $T_{\mathrm{e}} \# 6000 \mathrm{~K}$ qui correspond à la dissociation de $\mathrm{O}_{2}$ et l'autre à $T_{\mathrm{e}} \# 15000 \mathrm{~K}$ est lié à l'ionisation des espèces atomiques. Sa contribution dépasse $90 \%$ de $\lambda_{\mathrm{T}}$ à $T_{\mathrm{e}} \# 6000 \mathrm{~K}$.

- $\lambda_{\text {Int. }}$ reste faible sur toute la plage de température étudiée.

3.2.3.2 Conductibilité thermique totale. - Nous représentons la conductibilité thermique totale $\lambda_{\mathrm{T}}$, en fonction de la température $T_{\mathrm{e}}$ et pour $\theta=1 ; 1,5$; $2 ; 3 ; 4$, pour l'argon pur (Fig. 12) et pour le mélange à 


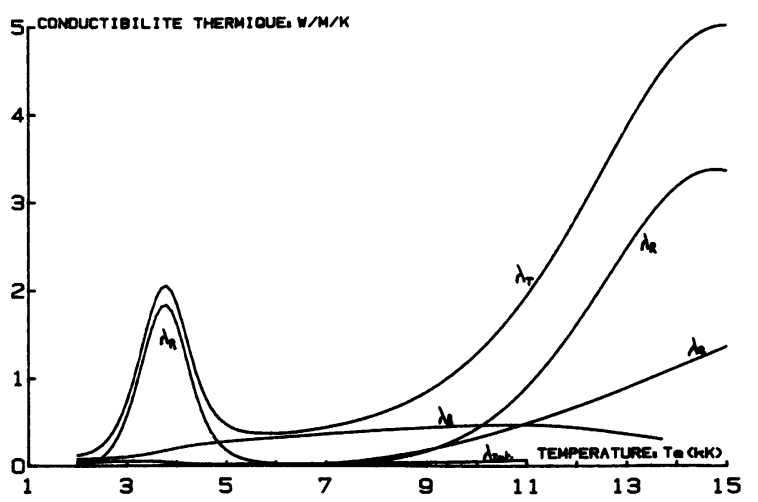

Fig. 11. - Evolution de la conductivité thermique totale $\lambda_{\mathrm{T}}$ ainsi que de ses quatre composantes : de translation des particules lourdes $\left(\lambda_{\mathrm{h}}\right)$, de translation des électrons $\left(\lambda_{\mathrm{e}}\right)$, de réaction $\left(\lambda_{R}\right)$ et interne $\left(\lambda_{\text {Inj. }}\right)$, en fonction de la température $T_{\mathrm{e}}$ et pour $\theta=2$, pour un plasma de mélange à $75 \%$ de $\mathrm{O}_{2}$ à la pression atmosphérique.

[Evolution, with electronic temperature, of the total thermal conductivity and of its four components for an argonoxygen plasma $\left(75 \%\right.$ of $\left.\mathrm{O}_{2}\right): p=1 \mathrm{~atm}$ and $\theta=2$.]

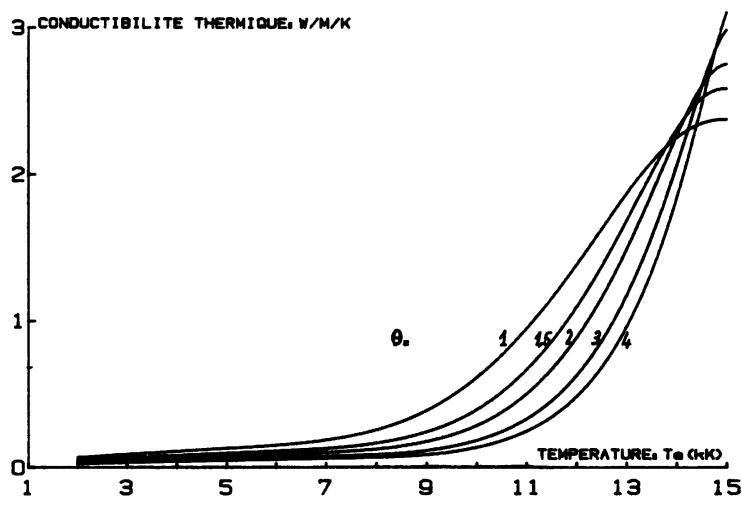

Fig. 12. - Evolution de la conductibilité thermique totale, en fonction de la température $T_{\mathrm{e}}$ et pour $\theta=1,1,5,2,3$ et 4 pour un plasma d'argon pur à la pression atmosphérique.

[Evolution, with electronic temperature and $\theta=1, \ldots, 4$, of the thermal conductivity for an argon plasma $(p=1 \mathrm{~atm})$.]

$50 \%$ d'oxygène (Fig. 15). Pour l'argon pur, nos résultats sont très différents de ceux publiés dans la littérature $[41,42]$ et ils sont notablement inférieurs pour $T_{\mathrm{e}}=15000 \mathrm{~K}$. Nous attribuons cet écart à l'approche théorique nouvelle de $\lambda_{\mathrm{h}}[10]$. Le tableau $\mathrm{V}$ compare, à $T_{\mathrm{e}}=15000 \mathrm{~K}$, nos résultats à ceux proposés par Kannapan et al. [41] et par Hsu et al. [42], pour la conductibilité thermique totale $\lambda_{\mathrm{T}}$ (exprimée en $\mathbf{W} / \mathrm{m} / \mathbf{K})$.

Pour le mélange à $50 \%$ d'oxygène (Fig. 13) nous trouvons, aux mêmes endroits que pour $\hat{C}_{p}$ (Fig. 6), les pics de dissociation de $\mathrm{O}_{2}\left(T_{\mathrm{e}} \# 4000 \mathrm{~K}\right.$ pour $\theta=1, T_{\mathrm{e}} \# 5000 \mathrm{~K}$ pour $\theta=1,5, T_{\mathrm{e}} \# 6000 \mathrm{~K}$ pour $\theta=2, T_{\mathrm{e}} \# 7500 \mathrm{~K}$ pour $\theta=3$ et $T_{\mathrm{e}} \# 8500 \mathrm{~K}$ pour $\theta=4$ ) et à plus hautes températures ceux d'ionisation des espèces atomiques.
Tableau V. - Comparaison des valeurs de la conductibilité thermique totale (exprimée en $\mathrm{W} / \mathrm{m} / \mathrm{K}$ ), à $T_{\mathrm{e}}=15000 \mathrm{~K}$, obtenus par Kannapan [41], Hsu [42] et nos résultats.

[Comparison between the total thermal conductivity values (in $\mathrm{W} / \mathrm{m} / \mathrm{K}$ ), at $T_{\mathrm{e}}=15000 \mathrm{~K}$, calculated by Kannapan [41], Hsu [42] and ours values.]

\begin{tabular}{|c|c|c|c|}
\hline$\theta$ & $\lambda_{\mathrm{T}}[41]$ & $\lambda_{\mathrm{T}}[42]$ & $\lambda_{\mathbf{T}} /$ Nos résultats \\
\hline- & - & - & - \\
\hline 1 & 2,4 & 2,4 & 2,38 \\
\hline & 15 & 5 & 2,76 \\
\hline & 76 & 9 & 2,99 \\
\hline & & $\sim 14$ & 3,11 \\
\hline
\end{tabular}

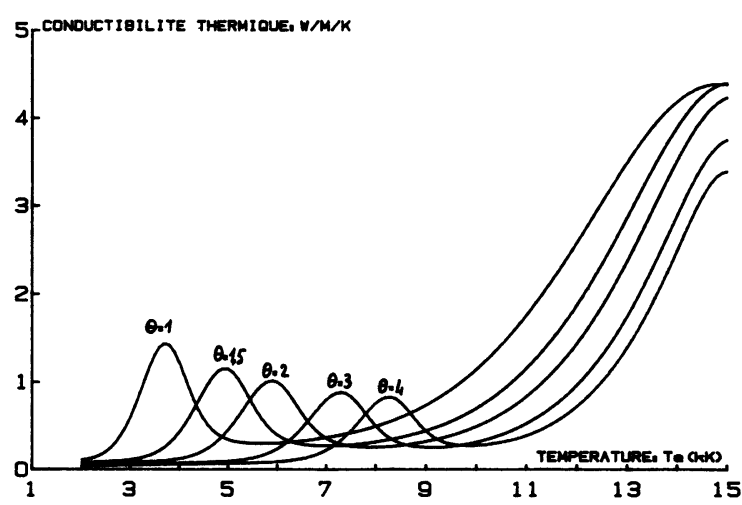

Fig. 13. - Evolution de la conductibilité thermique totale, en fonction de la température $T_{\mathrm{e}}$ et pour $\theta=1,1,5,2,3$ et 4 , pour un plasma de mélange à $50 \%$ de $\mathrm{O}_{2}$ à la pression atmosphérique.

[Evolution, with electronic temperature and $\theta=1, \ldots, 4$, of the total thermal conductivity for an argon-oxygen plasma $\left(50 \%\right.$ of $\left.\mathrm{O}_{2}\right): p=1 \mathrm{~atm}$.]

Finalement, sur la figure 14 nous représentons $\lambda_{\mathrm{T}}=f\left(T_{\mathrm{e}}\right)$ pour les deux séries d'intégrales de collision (note (1) et (2) pour l'interaction O-O, dans le cas de l'oxygène pur et à l'équilibre $(\theta=1)$. Dans le tableau VI, nous comparons nos valeurs de $\lambda_{\mathrm{T}}$, (en $\mathrm{W} / \mathrm{m} / \mathrm{K}$ ) avec celles de Krinberg et al. [47] qui sont toujours inférieurs aux nôtres. Il est raisonnable de penser que la valeur " réelle » de $\lambda_{\mathrm{T}}$ se situe entre les deux courbes référencées (1) et (2) sur la figure 14. En effet, les valeurs utilisées pour les intégrales de collision pour l'interaction $\mathrm{O}-\mathrm{O}$ correspondent aux deux extrêmes [48].

\section{Discussions.}

Dans la méthode MSE, lors du calcul de la composition du plasma, nous faisons l'hypothèse que la réaction de dissociation s'écrit :

$$
\mathrm{M}+\mathrm{O}_{2} \stackrel{k_{d}^{\gamma \mathrm{M}}}{\longrightarrow} \mathrm{M}+2 \mathrm{O} \quad E_{\mathrm{ac} .}^{\gamma} \# 5 \mathrm{eV} \text { [49] }
$$

où $\mathrm{M}$ représente une particule lourde $\left(\mathrm{M}=\mathrm{Ar}, \mathrm{O}_{2}, \mathrm{O}\right.$...) 


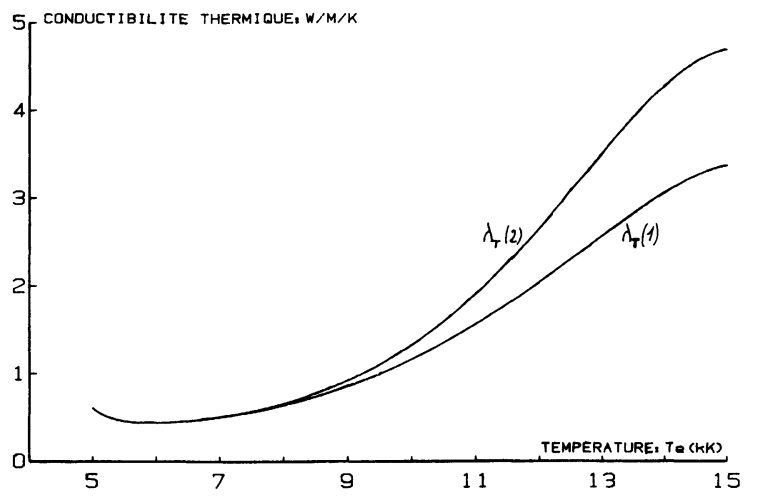

Fig. 14. - Evolution de la conductibilité thermique totale (en fonction de la température électronique $T_{\mathrm{e}}$, pour l'équilibre $(\theta=1)$ et pour un plasma d'oxygène pur à la pression atmosphérique obtenue à partir des données de Rutherford [35] (noté (1)) et de celles de Beebe [36] (noté (2)).

[Evolution, with electronic temperature of the total thermal conductivity calculated from Rutherford [35] (noted (1)) and the ones of Beebe [36] (noted (2)), for an oxygen plasma : $\theta=1$ and $p=1$ atm.

C'est-à-dire que nous négligeons la réaction :

$$
\mathrm{e}+\mathrm{O}_{2} \stackrel{k_{\hookrightarrow}^{\delta}}{\rightrightarrows} \mathrm{e}+2 \mathrm{O} \quad E_{\text {ac. }}^{\delta} \# 6 \mathrm{eV}[50]
$$

où $E_{\mathrm{ac} .}^{\gamma}$ et $E_{\mathrm{ac} .}^{\delta}$ sont les énergies d'activation, respectivement pour les réactions $\gamma$ et $\delta$.

Pour tester la validité de cette hypothèse, il faut comparer les vitesses de réaction pour la destruction de $\mathrm{O}_{2}$ :

$$
\sum_{\mathrm{M} \neq \mathrm{e}} n_{\mathrm{M}} n_{\mathrm{O}_{2}} k_{\mathrm{d}}^{\gamma, \mathrm{M}} \text { et } n_{\mathrm{e}} n_{\mathrm{O}_{2}} k_{\mathrm{d}}^{\delta} .
$$

L'important n'étant pas la valeur de ces vitesses mais plutôt leurs ordres de grandeur, nous écrivons, en première approximation :

$$
\left.\begin{array}{c}
k_{\mathrm{d}}^{\gamma, \mathrm{M}} \equiv k_{\mathrm{d}}^{\gamma} \# K \exp \left(-\frac{E_{\mathrm{ac} .}^{\gamma}}{k T_{\mathrm{h}}}\right) \\
k_{\mathrm{d}}^{\delta} \# K \exp \left(-\frac{E_{\mathrm{ac} .}^{\delta}}{k T_{\mathrm{e}}}\right) \\
\sum_{\mathrm{M} \neq \mathrm{e}} n_{\mathrm{M}} \# \sum_{i=1}^{N} n_{i}=n_{\mathrm{T}} .
\end{array}\right\}
$$

Compte tenu de ces trois relations, les vitesses de réactions s'écrivent alors : $n_{\mathrm{T}} n_{\mathrm{O}_{2}} k_{\mathrm{d}}^{\gamma}$ et $n_{\mathrm{e}} n_{\mathrm{O}_{2}} k_{\mathrm{d}}^{\delta}$ et nous calculons le rapport :

$$
R=\frac{n_{\mathrm{T}} n_{\mathrm{O}_{2}} k_{\mathrm{d}}^{\gamma}}{n_{\mathrm{e}} n_{\mathrm{O}_{2}} k_{\mathrm{d}}^{\delta}}=\frac{n_{\mathrm{T}}}{n_{\mathrm{e}}} \exp \left(-\frac{\theta E_{\mathrm{ac} .}^{\gamma}-E_{\mathrm{ac}}^{\delta}}{k T_{\mathrm{e}}}\right) .
$$

Dans le tableau VII, nous donnons les valeurs de ce rapport, calculer pour une température électronique
Tableau VI. - Comparaison des valeurs de la conductibilité thermique totale (exprimée en $\mathrm{W} / \mathrm{m} / \mathrm{K}$ ) : résultats de Krinberg [47] et les nôtres obtenus à partir des données de Rutherford [35] (noté 1) et de celles de Beebe [36] (noté 2).

[Comparison of the total thermal conductivity

$\begin{array}{rccl}T_{\mathrm{e}}(\mathrm{K}) & \lambda_{\mathrm{T}}(1) & \lambda_{\mathrm{T}}(2) & \lambda_{\mathrm{T}}[47] \\ - & - & - & - \\ 5000 & 0,600 & 0,600 & 0,550 \\ 10000 & 1,162 & 1,324 & 0,790 \\ 15000 & 3,367 & 4,688 & 2,90\end{array}$

Tableau VII. - Valeurs du rapport $R$ (relation (14)) permettant de tester la validité de la méthode MSE

\begin{tabular}{|c|c|c|c|c|}
\hline$\theta$ & $T_{\mathrm{e}}(\mathrm{K})$ & $n_{\mathrm{T}}\left(\operatorname{part} / \mathrm{m}^{3}\right)$ & $n_{\mathrm{e}}\left(\operatorname{part} / \mathrm{m}^{3}\right)$ & $R$ \\
\hline & 5000 & $2,2 \times 10^{24}$ & $3 \times \overline{1} 0^{18}$ & $10^{4}$ \\
\hline 2 & 6000 & $2,4 \times 10^{24}$ & $2 \times 10^{19}$ & 50 \\
\hline 3 & 7500 & $2,9 \times 10^{24}$ & $2 \times 10^{20}$ & 0,01 \\
\hline 4 & 8500 & $3,5 \times 10^{24}$ & $1 \times 10^{21}$ & $10^{-}$ \\
\hline
\end{tabular}
pour calculer la composition.

[Ratio values (relation (14)) which give the validity criteria for the MSE method.]

$T_{\mathrm{e}}$ correspondant au maximum du pic de dissociation pour $\hat{C}_{p}$ ou $\lambda_{\mathrm{T}}\left(T_{\mathrm{e}} \# 5000 \mathrm{~K}\right.$ pour $\theta=1,5 ; T_{\mathrm{e}} \# 6000 \mathrm{~K}$ pour $\theta=2 ; T_{\mathrm{e}} \# 7500 \mathrm{~K}$ pour $\theta=3$ et $T_{\mathrm{e}} \# 8500 \mathrm{~K}$ pour $\theta=4$ ).

L'hypothèse que nous faisons est donc réaliste que pour $\theta<3(R \gg 1)$. Une estimation plus précise de ces vitesses (utilisation des données des références $[49,50])$ conduit aux mêmes conclusions $(R=15$ pour $\theta=2$ et $T_{\mathrm{e}}=6000 \mathrm{~K} ; R=5 \times 10^{-3}$ pour $\theta=3$ et $\left.T_{\mathrm{e}}=7500 \mathrm{~K}\right)$.

Pour la détermination des propriétés de transport nous avons supposé que les sections efficaces (que nous calculons pour deux particules dans leur état fondamental) sont indépendantes du degré d'excitation des partenaires de collision. C'est-à-dire que les sections efficaces calculées, par exemple, pour l'interaction $0\left({ }^{3} \mathrm{P}\right)-0\left({ }^{3} \mathrm{P}\right)$ restent valables pour une collision faisant intervenir $0\left({ }^{3} \mathrm{P}\right)-0\left({ }^{1} \mathrm{D}\right), 0\left({ }^{3} \mathrm{P}\right)-0\left({ }^{1} \mathrm{~S}\right), 0\left({ }^{1} \mathrm{D}\right)$ $O\left({ }^{1} \mathrm{~S}\right), \ldots$ La prise en compte de toutes ces nouvelles intégrales modifierait nos résultats de moins de $10 \%$ à l'équilibre (cf. Capitelli [48]). L'approximation effectuée s'appuie sur deux justifications :

- Le choix entre deux jeux d'intégrales de collision (par exemple, dans notre cas, pour l'interaction $\mathrm{O}-\mathrm{O}^{+}$), entraîne des écarts sur les valeurs des propriétés de transport plus importantes (de l'ordre de $30 \%$ à $15000 \mathrm{~K}$, cf. Tableau VI) que l'approximation effectuée. 
- Il serait nécessaire de connaître avec une excellente précision les valeurs des densités numériques des différentes espèces du plasma. Or les approximations effectuées pour leur calcul sont parfois discutables (cf. début du paragraphe).

\section{Conclusion.}

Dans deux précédents articles $[9,10]$ nous avons établi un formalisme permettant d'obtenir les propriétés de transport en fonction des intégrales de collision définies par Hirschfelder [1], dans un plasma à deux « températures ». Nous retrouvons les résultats prévus par Devoto [2,3] à l'équilibre pour les processus élastiques sauf pour les coefficients de diffusion ordinaire [9] ce qui modifie le formalisme pour les processus inélastiques [10]. Dans ce travail nous avons appliqué ces résultats à un plasma de mélange $\mathrm{Ar}-\mathrm{O}_{2}$. Nous supposons le milieu composé des espèces suivantes $\mathrm{Ar}, \mathrm{Ar}^{+}, \mathrm{O}_{2}, \mathrm{O}_{2}^{+}, \mathrm{O}, \mathrm{O}^{+}, \mathrm{O}^{-}$et e, et nous passons en revue les potentiels d'interaction dont la connaissance est nécessaire au calcul des intégrales de collision. La composition du plasma est calculée à partir d'une méthode dérivée de celle de Potapov [11].

Les résultats obtenus pour l'argon pur sont en bon accord avec ceux publiés dans la littérature [41, 42] pour les propriétés de transport faisant intervenir les processus élastiques. Par contre, le nouveau développement que nous avons effectué pour les processus inélastiques $\left(\lambda_{\mathrm{R}}\right)$ modifie considérablement les valeurs de cette composante dont la contribution à la conductibilité thermique totale est importante. Il faut remarquer que dans cette nouvelle approche $\lambda_{\mathrm{T}}$ garde une valeur «finie" à $T_{\mathrm{e}}=15000 \mathrm{~K}$ (cf. Tableau V) ce qui n'est pas le cas pour les résultats proposés dans la littérature qui, pour $T_{\mathrm{e}}=15000 \mathrm{~K}$, n'ont plus grand sens physique et sont manifestement beaucoup trop grands. Par ailleurs, pour l'équilibre $(\theta=1)$ nos résultats sont en accord avec ceux obtenus à partir des théories classiques. Ceci constitue un bon test de validité de nos équations, $\theta=1$ n'étant pris que comme un cas particulier.

Le formalisme que nous avons développé permet, dans le principe, de déterminer les propriétés de transport hors équilibre mais il se heurte à deux difficultés. La première est liée à la détermination de la composition du plasma hors équilibre thermodynamique, détermination pour laquelle une méthode cinétique [39] ou des modèles de type collisionnel radiatif semblent mieux adaptés que ceux développés ici surtout pour les valeurs de $\theta \geqslant 3$. La deuxième difficulté réside dans le principe même de notre formalisme qui implique que l'écart à l'équilibre ne soit pas trop grand. Les résultats obtenus pour les valeurs de $\theta$ élevées $(\theta=3,4)$ doivent donc être considérés comme des indications de tendances et par conséquent pris avec précaution. Pour des plasmas fortement hors équilibre, il semblerait que des méthodes dérivées de celle proposée par Grad [51] soient mieux adaptées au problème.

\section{Remerciements.}

Les auteurs tiennent à remercier le professeur M. Capitelli pour ses conseils éclairés.

\section{Bibliographie}

[1] Hirschfelder, J. O., Curtis, C. F., Bird, R. B., Molecular Theory of Gases and Liquids (John Wiley and sons, N.Y.) 1964.

[2] Devoto, R. S., Phys. Fluids 9 (1966) 1230.

[3] Devoto, R. S., Phys. Fluids 10 (1967) 2105.

[4] Chapman, S., Cowling, T. G., The Mathematical Theory of Non-Uniform Gases (Cambridge University Press) 1970.

[5] Behsinger, K., Kollman, W., Mentel, J., Z. Phys. 215 (1968) 127.

[6] Kruger, C. H., Phys. Fluids 13 (1970) 1737.

[7] Coudert, J. F., Bourdin, E., Baronnet, J. M., Rakowitz, J., Fauchais, P., J. Physique Colloq. 40 (1979) C7-335.

[8] Shindoo, H., Inaba, T., Imazu, S., J. Phys. D 13 (1980) 805.

[9] Bonnefoi, C., Aubreton, J., Mexmain, J. M., FauChais, P., soumis Plasma Chem. Plasma Proc.

[10] Bonnefoi, C., Aubreton, J., Mexmain, J. M., accepté par Z. Natur. A.

[11] Potapov, A. V., High Temp. 4 (1966) 48.

[12] Aubreton, J., Bonnefor, C., Mexmain, J. M., soumis à Plasma Chem. Plasma Proc.
[13] Mason, E. A., Munn, R. J., Smith, F. J., Phys. Fluids 10 (1967) 1827.

[14] Devoto, R. S., Phys. Fluids 16 (1973) 616.

[15] Kihara, T., TAYloR, M. H., Hirschfelder, J. O., Phys. Fluids 3 (1960) 715.

[16] Monchick, L., Phys. Fluids 2 (1959) 695.

[17] Brokaw, R. S., Phys. Fluids 4 (1961) 944.

[18] Morse, P., Phys. Rev. 34 (1929) 57.

[19] Sмith, F. J., Munn, R. J., J. Chem. Phys. 41 (1961) 3560.

[20] Buckimgham, R. A., Proc. R. Soc. London A 168 (1938) 264.

[21] Mason, E. A., J. Chem. Phys. 22 (1954) 169.

[22] Parson, J. M., Siska, P. E., Lee, Y. T., J. Chem. Phys. 56 (1972) 1511.

[23] Luzzati, E., Pirani, F., Vecchiocattivi, F., Mol. Phys. 34 (1977) 1279.

[24] Dunning, T. H., HAG, P. J., J. Chem. Phys. 66 (1977) 3767.

[25] Brunetti, B., Liuti, G., Luzzati, E., Pirani, F., Vecchiocattivi, F., J. Chem. Phys. 74 (1981) 6734.

[26] Mexmain, J. M., Thèse 3e cycle, Limoges 1983. 
[27] Saxon, R., Liu, B., J. Chem. Phys. 67 (1977) 5432.

[28] Mason, E. A., Vanderslice, J. T., Yos, J. M., Phys. Fluids 6 (1959) 688.

[29] Dalgarno, A., Philos. Trans. R. Soc. London A 250 (1958) 426.

[30] Devoto, R. S., Phys. Fluids 10 (1967) 354.

[31] Moselay, J. T., Saxon, R. P., Huber, B. A., Cosby, P. C., Abouaf, R., Tadjeddine, M., J. Chem. Phys. 67 (1977) 1659.

[32] Michels, H. H., Hobbs, R. B., Wright, W. A., J. Chem. Phys. 69 (1978) 5151.

[33] Guest, M. F., Mol. Phys. 38 (1979) 1427.

[34] Moran, T. F., Flannerg, M. R., Cosby, P. C., J. Chem. Phys. 61 (1974) 1261.

[35] Rutherford, J. A., Vroom, D. A., J. Chem. Phys. 61 (1974) 2514.

[36] Beebe, N. H. F., Thulstrup, E. W., Andersen, J. Chem. Phys. 64 (1976) 2080.

[37] Itikawa, Y., At. Data Nucl. Data Tables 14 (1974) 1.

[38] Itikawa, Y., At. Data Nucl. Data Tables 21 (1978) 69.
[39] Richley, E., Tuma, D. T., J. Appl. Phys. 53 (1982) 8537.

[40] Cappitelli, M., Ficocelli, E., Molinari, E., Equilibrium Compositions and Thermodynamics properties of mixed plasma Ar- $\mathrm{O}_{2}$, Bari (1970).

[41] Kannapan, D., Bose, T. K., Phys. Fluids 16 (1973) 491.

[42] Hsu, K. C., Pfender, E., J. Appl. Phys. 54 (1983) 4359.

[43] Yos, J. M., RAD TF 65 (1965) 7.

[44] Vargafstik, N. B. (Hemisphere Publishing Corporation, London) 1975.

[45] Yun, K. S., Mason, E. A., Phys. Fluids 5 (1962) 380.

[46] Capitelli, M., Ficocelli, F., J. Phys. B 5 (1972) 2066.

[47] Krinberg, I. A., Tep. Vys. Temp. 3 (1965) 654.

[48] Capitelli, M., J. Physique Colloq. 38 (1977) C3-227.

[49] Jensen, D. E., Jones, G. A., Comb. Flame 32 (1978) 1.

[50] Fournier, F., Cinétique de l'Oxygène en Milieu Plasma, Ecole d'Eté des Houches (1983).

[51] Grad, H., Commun. Pure Appl. Math. 2 (1949) 33. 